\title{
Erratum to Andruff, Carraro, Thompson, Gaudreau, \& Louvet (2009): Latent Class Growth Modelling: A tutorial
}

\author{
Abstract: An error was found in Andruff, Carraro, Thompson, Gaudreau, \& Louvet \\ (2009). Here we identify the error and present a correction.
}

On page 14 of the original article, an error was made in the calculation of the average posterior probability of group membership for Trajectory 3 . The final sentence on this page should read "Returning to the hypothetical data set displayed in Table 1, the posterior probabilities of participants 4 and 6 with Trajectory 1 are averaged to obtain the average posterior probabilities of group membership for Trajectory $1[(0.93+1.00) \div 2=0.97]$ as are the posterior probabilities of participants 2 and 3 with Trajectory 2 [ $(1.00+.86) \div 2=0.93]$, and the posterior probabilities of participants 1 and 5 with Trajectory $3[(0.80+.76) \div 2=0.78] . "$

\section{References}

Andruff, H., Carraro, N., Thompson, A., Gaudreau, P., \& Louvet, B. (2009). Latent Class Growth Modelling: A tutorial. Tutorials in Quantitative Methods for Psychology, 5(1), 11-24.

The authors would like to thank D. S. Wood for noting the error in the original paper. 